\title{
Fault Detection and Isolation based on the Combination of a Bank of Interval Observers and Invariant Sets
}

\author{
Feng Xu, Florin Stoican, Vicenç Puig, Carlos Ocampo-Martinez and Sorin Olaru
}

\begin{abstract}
In this paper, a fault detection and isolation (FDI) approach using a bank of interval observers is developed. From the methodological point of view, a bank of interval observers is designed according to different dynamical models of the system under different modes (healthy or faulty). Each interval observer matches one system mode while all the interval observers monitor the system simultaneously. In order to guarantee FDI, a set of FDI conditions based on invariant set notions are established. These conditions ensure that the considered faults can be accurately isolated after a period of monitoring time. Finally, simulation results are used to present the effectiveness of the approach.
\end{abstract}

\section{INTRODUCTION}

According to the recent literature [2], [3], [5], interval observers have being successfully used for fault detection (FD) purposes, but not for fault isolation (FI) purposes. The aim of this present paper is to extend the use of interval observers to fault isolation (FI).

The interval observer-based FD approach consists in propagating the effect of uncertainties by means of the system mathematical models to generate adaptive thresholds for residuals. Then, the FD is performed by testing the consistency between model predictions and current measurements of the corresponding residuals [2], [5].

Whatever mode the system is under, an interval observer consistent with the current mode model of the system always predict state or output interval vectors that confine the current system states or outputs at each time instant. This mechanism provides useful information to detect and isolate faults when a bank of interval observers is used.

An FDI approach based on a bank of set-valued observersdifferent from interval observers-is proposed in [6]. Under a set of assumptions regarding the system, that proposed approach can implement FDI scheme. However, comparatively, the approach proposed in this paper provides a set of definite and pre-checkable FDI conditions that allow to know a priori whether faults are detectable and isolable.

Considering the good balance among expressional compactness, computational precision and complexity offered by zonotopes [1], this paper focuses on the representation

F. Xu, V. Puig and C. Ocampo-Martinez are with the Institut de Robòtica i Informàtica Industrial (CSIC-UPC), Technical University of Catalonia, Llorens i Artigas, 4-6, 08028 Barcelona, Spain, \{fxu, vpuig, cocampo\}@iri.upc.edu.

F. Stoican is with the Politehnica University of Bucharest (PUB) Department of Automatic Control and Systems Engineering, Bucharest, Romania, florin.stoican@acse.pub.ro.

S. Olaru is with the E3S (SUPELEC Systems Sceinces), Automatic Control Departement, Gif sur Yvette, France, sorin.olaru@supelec.fr. of uncertainties by zonotopes and the design of interval observers based on the Luenberger structure.

The contribution of this paper is twofold. First, it extends interval observer-based approaches to the case of FI, which implies that interval observers can independently implement FDI without the help of other FI techniques such as the fault signature matrices. Second, it establishes a set of FDI conditions to guarantee interval observer-based FDI.

The remainder of this paper is organized as follows. Section II introduces the notions of zonotopes and invariant sets. Section III introduces the plant and interval observers. The FDI algorithm is presented in Section IV. In Section $\mathrm{V}$, guaranteed FDI conditions are established. An extension of the approach for sensor faults is briefly discussed in Section VI. In Section VII, the examples are used to show the effectiveness of the proposed approach. In Section VIII, general conclusions are drawn.

\section{Preliminaries}

The notation $\oplus$ represents the Minkowski sum of two sets, |.| denotes the elementwise absolute value and the inequalities are interpreted elementwise.

\section{A. Invariant Sets}

The linear discrete time-invariant dynamics

$$
x_{k+1}=A_{\circ} x_{k}+B_{\circ} \delta_{k}
$$

are used to introduce the invariant set notions, where $A_{\circ}$ and $B_{\circ}$ are constant matrices and $A_{\circ}$ is a Schur matrix, $\delta_{k}$ belongs to $\Delta=\left\{\delta:\left|\delta-\delta^{\circ}\right| \leq \bar{\delta}\right\}$ with $\delta^{\circ}$ and $\bar{\delta}$ constant and all the elements have compatible dimensions.

Definition 2.1: (Invariant sets) A set $X \subset \mathbb{R}^{n}$ is called a robust $\lambda$-contractive (robust positively invariant $(R P I)$ ) set for (1) if and only if there exists a scalar $0 \leq \lambda<1(\lambda=1)$ such that $A_{\circ} X \oplus B_{\circ} \Delta \subseteq \lambda X$.

Definition 2.2: (The mRPI set) The minimal robust positively invariant set (mRPI set) with respect to (1) is defined as a RPI set contained in any closed RPI set.

Theorem 2.1: (Invariant sets) [4] Considering the dynamics (1) and letting $A_{\circ}=V \Lambda V^{-1}$ be the Jordan decomposition of $A_{\circ}$ with $\Lambda$ diagonal and $V$ invertible, the set

$$
\begin{aligned}
\Phi(\theta)= & \left\{x \in \mathbb{R}^{n}:\left|V^{-1} x\right| \leq(I-|\Lambda|)^{-1}\left|V^{-1} B_{\circ}\right| \bar{\delta}\right. \\
& +\theta\} \oplus \xi^{\circ}
\end{aligned}
$$

is RPI and attractive for the trajectories of (1), with $\theta$ any (arbitrarily small) vector with positive components, where $\xi^{\circ}$ is the center of the set that is expressed as $\xi^{\circ}=\left(I_{n}\right.$ $\left.A_{\circ}\right)^{-1} B_{\circ} \delta^{\circ}$ where $I_{n}$ is the identity matrix. 
1) For any $\theta$, the set $\Phi(\theta)$ is (positively) invariant, that is, if $x_{0} \in \Phi(\theta)$, then $x_{k} \in \Phi(\theta)$ for all $k \geq 0$.

2) Given $\theta \in \mathbb{R}^{n}, \theta>0$, and $x_{0} \in \mathbb{R}^{n}$, there exists $k^{*} \geq 0$ such that $x_{k} \in \Phi(\theta)$ for all $k \geq k^{*}$.

Remark 2.1: For $\theta>0$, the set $\Phi(\theta)$ is contractive. But for $\theta=0$, one can guarantee only the invariance and not the contractiveness of the set.

Proposition 2.1: (The mRPI set approximations) [4] Considering the dynamics (1), letting all eigenvalues of $A_{\circ}$ be strictly inside the unit circle and denoting $X_{0}$ as a (RPI) initial set of (1), each of the set iterations

$$
X_{j+1}=A_{\circ} X_{j} \oplus B_{\circ} \Delta, \quad j \in \mathbb{N},
$$

where $j$ denotes the $j$-th element in the set sequence and $\mathbb{N}$ represents the set of natural numbers, is a (RPI) approximation of the mRPI set of (1). Moreover, as $j$ tends to infinity, the set sequence converges to the mRPI set.

Remark 2.2: According to Theorem 2.1 and Proposition 2.1, one can compute a (RPI) approximation for the dynamics (1) with an arbitrarily expected approximate precision towards the mRPI set.

\section{B. Zonotopes}

According to [1] and [2], several definitions and properties of zonotopes are introduced.

Definition 2.3: (Zonotopes) Given a vector $p \in \mathbb{R}^{n}$ and a matrix $G \in \mathbb{R}^{n \times m}(n \leq m)$, a zonotope $X$ with the order $m$ is defined as $X=p \oplus G \mathbb{B}^{m}$.

Definition 2.4: (Interval hull) The interval hull $\square X$ of a zonotope $X=p \oplus G \mathbb{B}^{r} \subset \mathbb{R}^{n}$ is the smallest interval box that contains $X$ and the expression of the interval hull is denoted as $\square X=\left\{x|| x_{i}-p_{i} \mid \leq\left\|G_{i}\right\|_{1}\right\}$, where $G_{i}$ is the $i$-th row of $G$, and $x_{i}$ and $p_{i}$ are the $i$-th components of $x$ and $p$, respectively.

Property 2.1: (Minkowski sum) Given two zonotopes $X_{1}=p_{1} \oplus G_{1} \mathbb{B}^{r_{1}} \subset \mathbb{R}^{n}$ and $X_{2}=p_{2} \oplus G_{2} \mathbb{B}^{r_{2}} \subset \mathbb{R}^{n}$, the Minkowski sum of them is also a zonotope denoted by $X_{1} \oplus X_{2}=\left\{p_{1}+p_{2}\right\} \oplus\left[\begin{array}{ll}G_{1} & G_{2}\end{array}\right] \mathbb{B}^{r_{1}+r_{2}}$.

Property 2.2: (Mapping by a matrix) The image of a zonotope $X=p \oplus G \mathbb{B}^{r} \subset \mathbb{R}^{n}$ by a linear mapping matrix $K$ can be computed as $K X=K p \oplus K G \mathbb{B}^{r}$ by a standard matrix product.

Property 2.3: (Zonotope reordering) Given a zonotope $X=p \oplus G \mathbb{B}^{r} \subset \mathbb{R}^{n}$ and an integer $s$ (with $n<s<r$ ), denote by $\hat{G}$ the matrix resulting from the recording of the columns of the matrix $G$ in decreasing Euclidean norm. Then, $X \subseteq p \oplus\left[\begin{array}{ll}\hat{G}_{T} & Q\end{array}\right] \mathbb{B}^{s}$, where $\hat{G}_{T}$ is obtained from the first $s-n$ columns of matrix $\hat{G}$ and $Q \in \mathbb{R}^{n \times n}$ is a diagonal matrix that satisfies

$$
Q_{i i}=\sum_{j=s-n+1}^{r}\left|\hat{G}_{i j}\right|, i=1, \ldots, n .
$$

\section{Plant Models and Interval Observers}

In this section, the plant models with faults as well as interval observers are introduced.

\section{A. Plant Models}

The linear discrete time-invariant plant with faults is considered as

$$
\begin{aligned}
x_{k+1} & =A x_{k}+B F_{i_{a}} u_{k}+\omega_{k}, \\
y_{k} & =C G_{i_{s}} x_{k}+\eta_{k},
\end{aligned}
$$

where $x_{k} \in \mathbb{R}^{n}, u_{k} \in \mathbb{R}^{p}$ and $y_{k} \in \mathbb{R}^{q}$ are states, inputs and outputs, respectively, $A \in \mathbb{R}^{n \times n}, B \in \mathbb{R}^{n \times p}$ and $C \in \mathbb{R}^{q \times n}$ are constant, $F_{i_{a}} \in \mathbb{R}^{p \times p}\left(i_{a} \in \mathbb{I}_{a}=\{0,1,2, \ldots, N\}\right.$ where $N$ denotes the number of considered actuator faults) is a diagonal matrix modeling the $i_{a}$-th actuator mode, $F_{0}$ is the identity matrix representing the healthy actuator mode, $G_{i_{s}} \in \mathbb{R}^{n \times n}\left(i_{s} \in \mathbb{I}_{s}=\{0,1,2, \ldots, M\}\right.$ where $M$ denotes the number of considered sensor faults) is a diagonal matrix modeling the $i_{s}$-th sensor mode, $G_{0}$ is the identity matrix representing the healthy sensor mode, $\omega_{k} \in W$ and $\eta_{k} \in V$ represents bounded uncertainties (disturbances, offsets, etc) in states and outputs, respectively, and the subscript $k$ denotes the $k$-th discrete time ${ }^{1}$.

All diagonal entries of $F_{i_{a}}$ and $G_{i_{s}}$ belong to $[0,1]$ where 0 and 1 represent that the corresponding actuators/sensors are completely faulty or healthy, respectively, while a value in the range $(0,1)$ denotes a partial degradation of the corresponding actuators/sensors. $W$ and $V$ are defined as

$$
\begin{aligned}
W & =\left\{\omega_{k} \in \mathbb{R}^{n}:\left|\omega_{k}-\omega^{c}\right| \leq \bar{\omega}, \omega^{c} \in \mathbb{R}^{n}, \bar{\omega} \in \mathbb{R}^{n}\right\}, \\
V & =\left\{\eta_{k} \in \mathbb{R}^{q}:\left|\eta_{k}-\eta^{c}\right| \leq \bar{\eta}, \eta^{c} \in \mathbb{R}^{q}, \bar{\eta} \in \mathbb{R}^{q}\right\},
\end{aligned}
$$

where $\omega^{c}, \eta^{c}, \bar{\omega}$ and $\bar{\eta}$ are constant vectors.

Due to the structure above, $W$ and $V$ can be rewritten as zonotopes

$$
\begin{aligned}
W & =\omega^{c} \oplus H_{\bar{\omega}} \mathbb{B}^{n}, \\
V & =\eta^{c} \oplus H_{\bar{\eta}} \mathbb{B}^{q},
\end{aligned}
$$

where $\mathbb{B}^{n}$ and $\mathbb{B}^{q}$ are unitary boxes (interval vectors) composed of $n$ and $q$ unitary intervals, respectively, $H_{\bar{\omega}} \in \mathbb{R}^{n \times n}$ and $H_{\bar{\eta}} \in \mathbb{R}^{q \times q}$ are diagonal matrices with the diagonal entry in each row having the same value with the corresponding entry in each row of $\bar{\omega}$ and $\bar{\eta}$, respectively.

Assumption 3.1: (Fault occurrence) The system keeps operating in a dynamic mode for a sufficiently long time such that it goes into steady state before a switching of dynamic mode induced by a fault occurrence.

Assumption 3.2: (Detectability and stabilizability) The pairs $\left(A, B F_{i_{a}}\right)$ and $\left(A, C G_{i_{s}}\right)$ are respectively stabilizable and detectable under all the considered modes.

\section{B. Interval Observers}

1) The Notions of Interval Observers: Assuming that the plant (3) is in the healthy mode, an observer based on the Luenberger structure

$$
\begin{aligned}
\hat{x}_{k+1} & =A \hat{x}_{k}+B u_{k}+L_{0}\left(y_{k}-\hat{y}_{k}\right)+\check{\omega}_{k}, \\
\hat{y}_{k} & =C \hat{x}_{k}+\check{\eta}_{k},
\end{aligned}
$$

\footnotetext{
${ }^{1}$ Generally, sensor faults are modeled as $y_{k}=\check{G}_{i_{s}} C x_{k}+\eta_{k}$, but for simplicity of mathematical derivations, in this paper they are modeled as $y_{k}=C G_{i_{s}} x_{k}+\eta_{k}$.
} 
is designed, which includes uncertain variables $\check{\omega}_{k} \in W$ and $\check{\eta}_{k} \in V$, to model the effect of unknown disturbances $\omega_{k}$ and noises $\eta_{k}$ in the real states $x_{k}$ and outputs $y_{k}$ of the plant, respectively, and where $L_{0}$ is an observer gain matrix that can ensure the observer convergence.

By introducing zonotope description of noises and disturbances as indicated in (4a) and (4b) into the observer mapping (5) and using zonotope arithmetic at each time instant [1], a healthy interval observer based on the Luenberger structure is given as

$$
\begin{aligned}
\hat{X}_{k+1}^{0}= & \left(A-L_{0} C\right) \hat{X}_{k}^{0} \oplus\left\{B u_{k}\right\} \oplus\left\{L_{0} y_{k}\right\} \\
& \oplus\left(-L_{0}\right) V \oplus W, \\
\hat{Y}_{k}^{0}= & C \hat{X}_{k}^{0} \oplus V,
\end{aligned}
$$

where $\hat{X}_{k}^{0}$ and $\hat{Y}_{k}^{0}$ are respectively state and output zonotopes predicted by the interval observer at time instant $k$. Eventually, it is guaranteed that the predicted zonotopes for $\hat{y}_{k}$ (or $\hat{x}_{k}$ ) confine both $y_{k}$ (or $x_{k}$ ) and $\hat{y}_{k}$ (or $\hat{x}_{k}$ ), respectively.

2) Interval Observers for Actuator Faults: In (3), letting $G_{i_{s}}$ be the identity matrix, one can obtain the models of the plant under actuator faults. The interval observer corresponding to the model of the $j_{a}$-th system actuator mode is designed as

$$
\begin{aligned}
\hat{X}_{k+1}^{j_{a}=} & \left(A-L_{j_{a}} C\right) \hat{X}_{k}^{j_{a}} \oplus\left\{B F_{j_{a}} u_{k}\right\} \oplus\left\{L_{j_{a}} y_{k}\right\} \\
& \oplus\left(-L_{j_{a}}\right) V \oplus W, \\
\hat{Y}_{k}^{j_{a}}= & C \hat{X}_{k}^{j_{a}} \oplus V,
\end{aligned}
$$

where $j_{a} \in \mathbb{I}_{a}$ represents the index of the interval observer $\left(j_{a}=0\right.$ denotes the healthy interval observer as seen in (6)), $\hat{X}_{k}^{j_{a}}$ and $\hat{Y}_{k}^{j_{a}}$ are state and output zonotopes predicted by the $j_{a}$-th interval observer at $k$, respectively, and $L_{j_{a}}$ is an observer gain matrix which makes $A-L_{j_{a}}$ be a Schur matrix.

According to (7) and zonotope operations, the center $\hat{x}_{k+1}^{c, j_{a}}$ and segment matrix $\hat{H}_{k+1}^{j_{a}}$ of $\hat{X}_{k+1}^{j_{a}}$, and the center $\hat{y}_{k}^{c, j_{a}}$ and segment matrix $\hat{H}_{k}^{j_{a}}$ of $\hat{Y}_{k}^{j_{a}}$ are computed as

$$
\begin{aligned}
\hat{x}_{k+1}^{c, j_{a}}= & \left(A-L_{j_{a}} C\right) \hat{x}_{k}^{c, j_{a}}+B F_{j_{a}} u_{k}+L_{j_{a}} y_{k} \\
& -L_{j_{a}} \eta^{c}+w^{c}, \\
\hat{H}_{k+1}^{j_{a}}= & {\left[\left(A-L_{j_{a}} C\right) \hat{H}_{k}^{j_{a}}-L_{j_{a}} H_{\bar{\eta}} H_{\bar{\omega}}\right], } \\
\hat{y}_{k}^{c, j_{a}}= & C \hat{x}_{k}^{c, j_{a}}+\eta^{c}, \\
\hat{H}_{k}^{j_{a}}= & {\left[C \hat{H}_{k}^{j_{a}} H_{\bar{\eta}}\right] . }
\end{aligned}
$$

3) Interval Observers for Sensor Faults: Similarly, in (3) letting $F_{i_{a}}$ be the identity matrix, the models of the plant under sensor faults can be obtained. The interval observer corresponding to the $j_{s}$ th system sensor mode can be expressed as

$$
\begin{aligned}
\hat{X}_{k+1}^{j_{s}}= & \left(A-L_{j_{s}} C G_{j_{s}}\right) \hat{X}_{k}^{j_{s}} \oplus\left\{B u_{k}\right\} \oplus\left\{L_{j_{s}} y_{k}\right\} \\
& \oplus\left(-L_{j_{s}}\right) V \oplus W, \\
\hat{Y}_{k}^{j_{s}}= & C G_{j_{s}} \hat{X}_{k}^{j_{s}} \oplus V
\end{aligned}
$$

where $j_{s} \in \mathbb{I}_{s}$ represents the index of the interval observer ( $j_{s}=0$ denotes the healthy interval observer as seen in (6)) and the gain matrix $L_{j_{s}}$ can make $A-L_{j_{s}} C G_{j_{s}}$ be a Schur matrix. Similar with (8), the expressions of the center and segment matrix of $\hat{X}_{k+1}^{j_{s}}$ and $\hat{Y}_{k}^{j_{s}}$ can be derived.

Assumption 3.3: (Initial conditions) The initial state of the plant is denoted as $x_{0}$ and $x_{0}$ belongs to a known initial zonotope $\hat{X}_{0}$ for all the interval observers, i.e., $x_{0} \in \hat{X}_{0}$.

Since the prediction of interval observers and the computation of interval vectors is based on zonotopes, the discussions in the remaining of the paper are mainly based on zonotopes.

Additionally, since the principle of the proposed technique for actuator and sensor FDI is similar, in this paper only FDI of actuator faults is discussed in detail. However, the extension of the method to the case of sensor faults is summarized afterwards.

\section{Proposed Interval Observer-BAsed FDI}

The interval observer-based FDI is introduced and the proposed FDI algorithm is presented.

\section{A. FDI using Interval Observers}

1) FD using Interval Observers: According to [3], the interval observer-based FD uses the heathy interval observer, which is based on propagating model uncertainties to the residuals and checking if

$$
\mathbf{0} \in \square R_{k}^{0},
$$

where $R_{k}^{0}=\left\{y_{k}\right\} \oplus\left(-\hat{Y}_{k}^{0}\right)$ denotes the residual zonotope predicted by the healthy interval observer at time instant $k$ and 0 represents the zero vector. If (10) does not hold, it is assumed that a fault has occurred at $k$.

2) FI using Interval Observers: The proposed FI technique is based on a bank of interval observers and each observer is designed to match a given system mode. At each time instant, a set of residual zonotopes predicted by the bank of interval observers can be obtained. After the transition from one operating mode to another, the residual zonotope matching the current mode should include $\mathbf{0}$ and simultaneously all the other residual zonotopes not matching the current mode should always exclude $\mathbf{0}$.

\section{B. FDI Algorithm using a Bank of Interval Observers}

Since each interval observer matches one certain system mode, it means that each interval observer has different dynamical behaviors under different modes. Since a fault occurrence always induces the corresponding uncertainties on dynamical behaviors of interval observers during the transition, there exist possibilities that at some time instants several residual zonotopes predicted by several different interval observers simultaneously contain $\mathbf{0}$ during the transition.

In order to guarantee the correct and timely FI, a waiting time $T$ is necessary after a fault is detected. This waiting time is used to delay FI process such that the incorrect FI possibilities are completely avoided. The procedure of this proposed FDI method is presented in Algorithm 1.

Definition 4.1: (Waiting time T) It is defined as, at least, the maximum of all the settling time of all the interval observers such that residual zonotopes predicted by interval 
observes not matching the current system mode do exclude $\mathbf{0}$ by waiting $T$ after the detection of a fault.

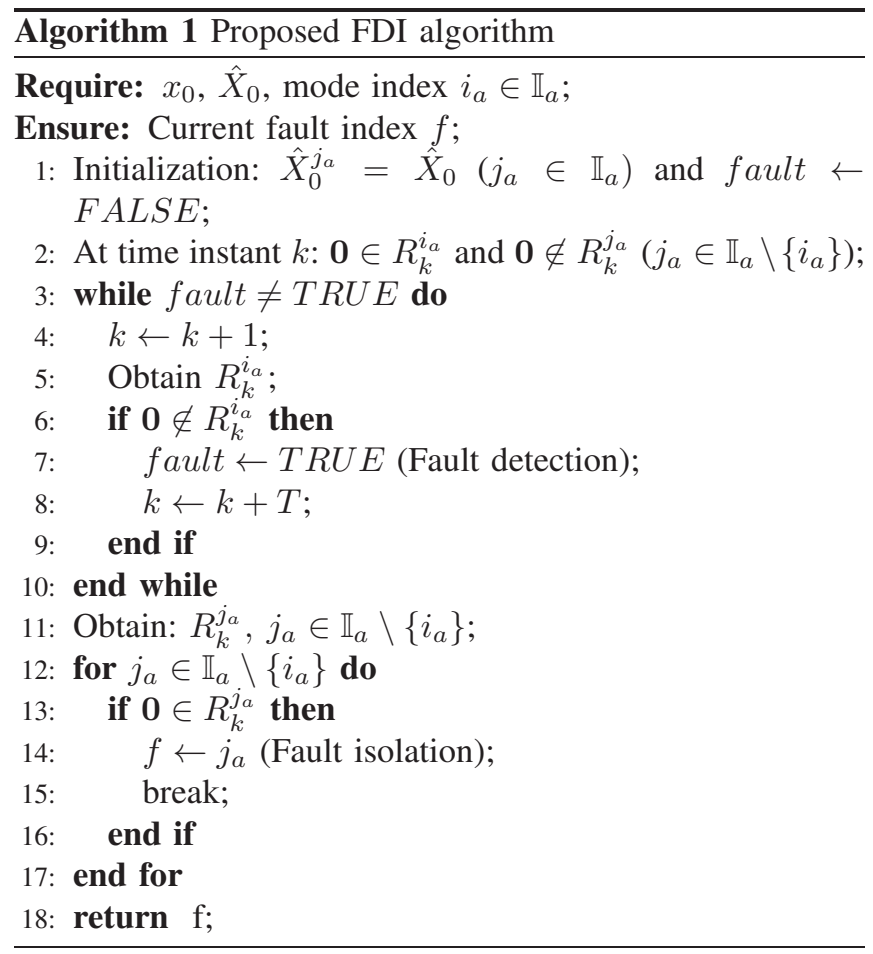

Remark 4.1: When a fault occurs, it always results in changes in the system outputs (or the inputs of interval observers) and induces a transition for the estimations of each interval observer. Theoretically, the transition is assessed by the observer settling time, i.e., the eigenvalues of the interval observer matrix. Thus, by adjusting observer gain matrices of all the interval observers, one can obtain a satisfactory waiting time to guarantee reliable FDI. However, this adjustment should depend on the particular applications.

\section{Guaranteed FDi Conditions}

This section establishes a set of FDI sufficient conditions based on a bank of interval observers.

\section{A. Characterizing Residual Sets using Zonotopes}

When the system is under the $i_{a}$-th actuator mode, the residual zonotopes predicted by the $j_{a}$-th interval observer is defined as

$$
\begin{aligned}
R_{k}^{i_{a} j_{a}} & =\left\{y_{k}\right\} \oplus\left(-\hat{Y}_{k}^{j_{a}}\right) \\
& =\left\{C x_{k}+\eta_{k}\right\} \oplus\left\{\left(-C \hat{X}_{k}^{j_{a}}\right) \oplus(-V)\right\} \\
& =C\left\{\left\{x_{k}\right\} \oplus\left(-\hat{X}_{k}^{j_{a}}\right)\right\} \oplus\left\{\eta_{k}\right\} \oplus(-V) .
\end{aligned}
$$

In order to describe the residual zonotopes defined in (11), one has to obtain $\tilde{X}_{k}^{i_{a} j_{a}}$ which is written as

$$
\begin{aligned}
\tilde{X}_{k}^{i_{a} j_{a}} & =\left\{x_{k}\right\} \oplus\left(-\hat{X}_{k}^{j_{a}}\right) \\
& =\left\{\left(x_{k}-\hat{x}_{k}^{c, j_{a}}\right)\right\} \oplus \hat{H}_{k}^{j_{a}} \mathbb{B}^{s_{k}^{j_{a}}} \\
& =\tilde{x}_{k}^{c, i_{a} j_{a}} \oplus \tilde{H}_{k}^{i_{a} j_{a}} \mathbb{B}^{s_{k}^{j_{a}}}
\end{aligned}
$$

where $\tilde{x}_{k}^{c, i_{a} j_{a}}=x_{k}-\hat{x}_{k}^{c, j_{a}}, \tilde{H}_{k}^{i_{a} j_{a}}=\hat{H}_{k}^{j_{a}}$ and $s_{k}^{j_{a}}$ represents the order of the zonotope $\hat{X}_{k}^{j_{a}}$.

According to (3) and (8), the center and segment matrix of $\tilde{X}_{k+1}^{i_{a} j_{a}}$ are computed as

$$
\begin{aligned}
\tilde{x}_{k+1}^{c, i_{a} j_{a}}= & \left(A-L_{j_{a}} C\right) \tilde{x}_{k}^{c, i_{a} j_{a}}+B\left(F_{i_{a}}-F_{j_{a}}\right) u_{k} \\
& -L_{j_{a}}\left(\eta_{k}-\eta^{c}\right)+\left(\omega_{k}-\omega^{c}\right), \\
\tilde{H}_{k+1}^{i_{a} j_{a}}= & \hat{H}_{k+1}^{j_{a}}=\left[\left(A-L_{j_{a}} C\right) \hat{H}_{k}^{j_{a}}-L_{j_{a}} H_{\bar{\eta}} H_{\bar{\omega}}\right] .
\end{aligned}
$$

In order to establish guaranteed FDI conditions, assume that all possible values of control inputs $u_{k}$ belong to a set denoted as

$$
U=\left\{u_{k} \in \mathbb{R}^{p}:\left|u_{k}-u^{c}\right| \leq \bar{u}, u^{c} \in \mathbb{R}^{p}, \bar{u} \in \mathbb{R}^{p}\right\},
$$

where $u^{c}$ and $\bar{u}$ are constant. Moreover, $U$ can be rewritten as a zonotope

$$
U=u^{c} \oplus H_{\bar{u}} \mathbb{B}^{p}
$$

where $H_{\bar{u}} \in \mathbb{R}^{p \times p}$ is a diagonal matrix with the diagonal entry in each row having the same value with the corresponding entry in the same row of $\bar{u}$.

By substituting $U, W$ and $V$ to replace $u_{k}, \omega_{k}$ and $\eta_{k}$ in (13a), respectively, one can compute a bounding zonotope denoted as $\breve{X}_{k+1}^{i_{a} j_{a}}$ to bound $\tilde{X}_{k+1}^{i_{a} j_{a}}$ at time instant $k+1$, and the center and segment matrix of $\breve{X}_{k+1}^{i_{a} j_{a}}$ are derived as

$$
\begin{aligned}
\breve{x}_{k+1}^{c, i_{a} j_{a}}= & \left(A-L_{j_{a}} C\right) \breve{x}_{k}^{c, i_{a} j_{a}}+B\left(F_{i_{a}}-F_{j_{a}}\right) u^{c}, \\
\breve{H}_{k+1}^{i_{a} j_{a}}= & {\left[\left(A-L_{j_{a}} C\right) \hat{H}_{k}^{j_{a}} B\left(F_{i_{a}}-F_{j_{a}}\right) H_{u}-L_{j_{a}} H_{\bar{\eta}}\right.} \\
& \left.L_{j_{a}} H_{\bar{\eta}} H_{\bar{\omega}}-H_{\bar{\omega}}\right] .
\end{aligned}
$$

Comparing (13) with (14), it is seen that as long as the dynamics of $\tilde{X}_{k+1}^{i_{a} j_{a}}$ and $\tilde{X}_{k+1}^{i_{a} j_{a}}$ are initialized under the condition $\tilde{X}_{0}^{i_{a} j_{a}} \subseteq \widetilde{X}_{0}^{i_{a} j_{a}}$, after the initialization $\tilde{X}_{k+1}^{i_{a} j_{a}} \subseteq$ $\breve{X}_{k+1}^{i_{a} j_{a}}$ holds for all $k>0$.

Thus, one can obtain the set-based dynamics of (14), which is derived as

$$
\begin{aligned}
\breve{X}_{k+1}^{i_{a} j_{a}}= & \left(A-L_{j_{a}} C\right) \breve{X}_{k}^{i_{a} j_{a}} \oplus B\left(F_{i_{a}}-F_{j_{a}}\right) U \oplus L_{j_{a}}(-V) \\
& \oplus W \oplus L_{j_{a}} V \oplus(-W) .
\end{aligned}
$$

In order to establish a set of guaranteed FDI conditions, this paper is interested in $\breve{X}_{\infty}^{i_{a} j_{a}}$ at infinity. In fact, it is not possible to accurately compute $X_{\infty}^{i_{a} j_{a}}$. Then, one has to compute an approximation for $\breve{X}_{\infty}^{i_{a} j_{a}}$ and as long as the precision of the approximation is satisfactory, it can be used to replace the use of $\breve{X}_{\infty}^{i_{a} j_{a}}$.

By following Theorem 2.1 and Proposition 2.1, assigning an arbitrarily initial zonotope ${ }^{2}$ for (15) and iterating (15), a satisfactory approximation of $\breve{X}_{\infty}^{i_{a} j_{a}}$ denoted as $S_{i_{a} j_{a}}$ with the center $O_{i_{a} j_{a}}$ can be obtained.

\footnotetext{
${ }^{2}$ Note that according to Theorem 2.1 a RPI set of (15) can be obtained. Thus, if the initial zonotope is RPI, it is guaranteed that $S_{i_{a} j_{a}}$ is a RPI approximation of $\breve{X}_{\infty}^{i_{a} j_{a}}$. if the initial is not RPI, a non-RPI approximation for $\breve{X}_{\infty}^{i_{a} j_{a}}$ can be obtained. However, as long as the iterative time is sufficient, the non-RPI approximation can also be satisfactory.
} 


\section{B. Guaranteed FDI Conditions}

For each considered system mode, an interval observer is designed to match the corresponding mode. According to (11) and (12), the residual zonotope at time instant $k$ is rewritten as

$$
R_{k}^{i_{a} j_{a}}=C \tilde{X}_{k}^{i_{a} j_{a}} \oplus\left\{\eta_{k}\right\} \oplus(-V) .
$$

By substituting $V$ to replace $\eta_{k}$ in (16), a residualbounding zonotope $\breve{R}_{k}^{i_{a} j_{a}}$ at $k$ can be obtained as

$$
\breve{R}_{k}^{i_{a} j_{a}}=C \breve{X}_{k}^{i_{a} j_{a}} \oplus V \oplus(-V) .
$$

As $k$ tends to infinity, guaranteed FDI sufficient conditions based on $\breve{R}_{\infty}^{i_{a} j_{a}}$ can be established.

Theorem 5.1: (Guaranteed FDI conditions) Considering the plant (3) and a bank of interval observers (7), as long as the residual-bounding zonotope $\breve{R}_{\infty}^{i_{a} j_{a}}\left(j_{a} \neq i_{a}\right.$ and $i_{a}$, $j_{a} \in \mathbb{I}_{a}$ ) satisfies

$$
\left|P_{l}\left(\mathbf{0}-\breve{r}_{i_{a} j_{a}}^{c}\right)\right|>\max _{r \in E}\left|P_{l}\left(r-\breve{r}_{i_{a} j_{a}}^{c}\right)\right|,
$$

where $\breve{r}_{i_{a} j_{a}}^{c}$ denotes the center of $\breve{R}_{\infty}^{i_{a} j_{a}}, \breve{E}$ represents the set of all vertices of $\breve{R}_{\infty}^{i_{a} j_{a}}, P_{l}($.$) represents the projection$ towards the axis $l \in\{1,2, \ldots, q\}$, once a fault occurs, the accurate FDI can be guaranteed after a waiting time.

Proof : The proof has two parts. The first part is to prove that $\breve{R}_{\infty}^{i_{a} j_{a}}\left(j_{a} \neq i_{a}\right)$ does not contain $\mathbf{0}$, which is the asymptotic FDI condition. The second part concentrates on that the dynamical behavior of the residuals at infinity $\left(\breve{R}_{\infty}^{i_{a} j_{a}}\right)$ translates those after a waiting time, which guarantees the FDI reliability and accuracy.

The satisfaction of (18) implies that the limit set $\breve{R}_{\infty}^{i j_{a}}$ does not contain 0 . Thus, one only focuses on the proof of the second part as follows. Since residual zonotopes and their bounding zonotopes are determined by (13) and (14), without loss of effectiveness, the main elements used next will be these set-based dynamics.

The equation (15) shows that the time-variant term is $(A-$ $\left.L_{j_{a}} C\right) \breve{X}_{k}^{i_{a} j_{a}}$, which means that the difference of values of $\breve{X}_{k}^{i_{a} j_{a}}$ at different time instants is determined by the shape of $\breve{X}_{0}^{i_{a} j_{a}}$, while the contractive factor is determined by the placement of the eigenvalues of $A-L_{j_{a}} C$ that corresponds to the $j_{a}$-th interval observer.

Thus, whenever a fault occurs, after a waiting time assessed by the eigenvalues of the interval observer, (15) enters into steady state. Then, the set value of $\breve{X}_{k}^{i_{a} j_{a}}$ can be sufficiently ${ }^{3}$ close to that of $\breve{X}_{\infty}^{i_{a} j_{a}}$, which means that $\breve{X}_{\infty}^{i_{a} j_{a}}$ can approximately describe the dynamical behaviors of the system after the waiting time.

As long as Theorem 5.1 is satisfied, the FDI of any of considered faults can be guaranteed. However, since $\breve{R}_{\infty}^{i_{a} j_{a}}$ can not be accurately computed but only approximated, Theorem 5.1 has only a theoretical value. For the sake of finding a set of practical FDI conditions, one has to turn

\footnotetext{
${ }^{3} \breve{X}_{k}^{i_{a} j_{a}}$ is inside the set described as the Minkowski sum of $\left\{P_{i_{a} j_{a}}\right\} \oplus$ $(1+\epsilon)\left\{\breve{X}_{\infty}^{i_{a} j_{a}} \oplus\left\{-P_{i_{a} j_{a}}\right\}\right\}$, where $P_{i_{a} j_{a}}$ denotes the center of $\breve{X}_{\infty}^{i_{a} j_{a}}$ and $\epsilon$ is a scalar that satisfies $\epsilon>0$.
}

to an approximation $S_{i_{a} j_{a}}$ of $\breve{R}_{\infty}^{i j}$. Further, a satisfactory approximation of $\breve{R}_{\infty}^{i_{a} j_{a}}$ is derived as

$$
\stackrel{\circ}{R}_{\infty}^{i_{a} j_{a}}=C S_{i_{a} j_{a}} \oplus V \oplus(-V),
$$

where the center of $\stackrel{\circ}{R}_{\infty}^{i_{a} j_{a}}$ is computed as

$$
{\stackrel{\circ}{r_{a} j_{a}}}^{c}=C O_{i_{a} j_{a}} .
$$

Based on (19), (20) and Theorem 5.1, a set of usable FDI conditions can be established

$$
\left|P_{l}\left(\mathbf{0}-{\stackrel{r}{i_{a} j_{a}}}^{c}\right)\right|>\max _{r \in E}\left|P_{l}\left(r-{\stackrel{r}{i_{a} j_{a}}}^{c}\right)\right|,
$$

where $\stackrel{\circ}{E}$ represents the set of all vertices of ${\stackrel{\circ}{R_{\infty}}}_{\infty}^{i_{a} j_{a}}\left(j_{a} \neq i_{a}\right)$.

Note that the guaranteed FDI conditions are a set of sufficient conditions, not necessary conditions due to the series of approximations contained in the design method. Thus, their satisfaction can guarantee FDI, but the dissatisfaction does not mean that the faults are non-detectable or non-isolable with extra effort.

\section{The Extension for Sensor Faults}

When the plant (3) is under a sensor fault, similarly, residual zonotopes predicted by the $j_{s}$-th interval observers under the $i_{s}$-th system mode can be derived as

$$
\begin{aligned}
R_{k}^{i_{s} j_{s}} & =\left\{y_{k}\right\} \oplus\left(-\hat{Y}_{k}^{j_{s}}\right) \\
& =\left\{C G_{i_{s}} x_{k}+\eta_{k}\right\} \oplus\left\{\left(-C G_{j_{s}} \hat{X}_{k}^{j_{s}}\right) \oplus(-V)\right\} \\
& =\left\{C G_{i_{s}} x_{k}\right\} \oplus\left(-C G_{j_{s}} \hat{X}_{k}^{j_{s}}\right) \oplus\left\{\eta_{k}\right\} \oplus(-V) .
\end{aligned}
$$

In order to establish a set of sensor FDI conditions like (18) in the case of actuator faults, in the $i_{s}$-th mode one has to compute the corresponding bounding zonotope $\breve{R}_{k}^{i_{s} j_{s}}$ to bound $R_{k}^{i_{s} j_{s}}$ at each time instant. According to (22), one further has

$$
\begin{aligned}
R_{k}^{i_{s} j_{s}} \subseteq & C G_{i_{s}}\left\{\left\{x_{k}\right\} \oplus\left(-\hat{X}_{k}^{j_{s}}\right)\right\} \oplus C\left(G_{i_{s}}-G_{j_{s}}\right) \hat{X}_{k}^{j_{s}} \\
& \oplus\left\{\eta_{k}\right\} \oplus(-V) .
\end{aligned}
$$

As discussed in the previous sections, the bounding zonotopes of $\left\{x_{k}\right\} \oplus\left(-\hat{X}_{k}^{j_{s}}\right)$ and $\hat{X}_{k}^{j_{s}}$, respectively denoted as $\breve{X}_{k}^{i_{s} j_{s}}$ and $\hat{X}_{k}^{j_{s}}$, can be computed in the same way. Thus, $\breve{R}_{k}^{i_{s} j_{s}}$ to bound $R_{k}^{i_{s} j_{s}}$ can be derived as

$$
\breve{R}_{k}^{i_{s} j_{s}}=C G_{i_{s}} \breve{X}_{k}^{i_{s} j_{s}} \oplus C\left(G_{i_{s}}-G_{j_{s}}\right) \breve{\hat{X}}_{k}^{j_{s}} \oplus V \oplus(-V) .
$$

Similarly, by obtaining satisfactory approximations of $\breve{X}_{\infty}^{i_{s} j_{s}}$ and $\breve{X}_{\infty}^{j_{s}}$, the corresponding approximation ${\stackrel{\circ}{R^{i}}}^{i_{s} j_{s}}$ for $\breve{R}_{\infty}^{i_{s} j_{s}}$ can be obtained. Thus, based on the same principle with the case of actuator faults, a set of guaranteed FDI conditions can be established for sensor FDI.

However, from the derivation indicated in (23), it is shown that the proposed method for sensor FDI is conservative. Thus, in this paper the discussions are restricted to this remark. Note that, if a less conservative method can be found, the conservativeness of guaranteed FDI conditions for sensor faults will be further reduced, which will be an important point of our further research. 


\section{NuMERICAL EXAMPLE}

The dynamics of the second blade subsystem of a wind turbine benchmark indicated in [7] are used for the illustrative example. Considering the length of this paper, please refer to Eqs.(4), (5) and (6) in [7] for the details of the subsystem dynamics structure.

1) The Case of Actuator Faults: We assume that the dynamics have two actuator-fault modes, i.e, the dynamics Eq.(4) in [7] are rewritten as

$$
\begin{aligned}
x_{\beta_{2}}^{+} & =A_{\beta_{2}} x_{\beta_{2}}+B_{\beta_{2}} F_{i_{a}}\left(\beta_{r}+\beta_{2 f}\right), \\
\beta_{2} & =C_{\beta_{2}} x_{\beta_{2}},
\end{aligned}
$$

where the notation + , consistent with [7] for simplicity, denotes the successor time instant, $F_{i_{a}}$ models the $i_{a}$-th actuator mode $\left(i_{a} \in\{0,1,2\}\right)$ and $F_{0}$, the identity matrix, represents the healthy actuator mode.

We assume that the two sensors of the subsystem are healthy (i.e., $K=1$ in Eq.(5) of [7]) and that the feedback $\beta_{2 f}$ is obtainable. Three interval observers are designed as indicated in (7) corresponding to the three modes. After system discretization with the sampling time $0.01 \mathrm{~s}$, the parameters of the discrete-time dynamics are given as

- model parameters:

$$
\begin{aligned}
& A_{\beta_{2}}=\left[\begin{array}{cc}
0.8667 & -1.2343 \\
0.01 & 1
\end{array}\right], B_{\beta_{2}}=\left[\begin{array}{c}
0.01 \\
0
\end{array}\right], \\
& C_{\beta_{2}}=\left[\begin{array}{ll}
0 & 123.4321
\end{array}\right],
\end{aligned}
$$

- measurement noises:

$$
\begin{aligned}
& \bar{\eta}_{\beta_{2}, m 1}=0.03, \quad \eta_{\beta_{2}, m 1}^{c}=0.3, \\
& \bar{\eta}_{\beta_{2}, m 2}=0.03, \quad \eta_{\beta_{2}, m 2}^{c}=0.3,
\end{aligned}
$$

- Three observer gains:

$$
L_{0}=L_{1}=L_{2}=\left[\begin{array}{c}
-0.001 \\
0.003
\end{array}\right],
$$

- fault magnitude:

$$
F_{1}=[0.1], F_{2}=[0.5],
$$

- sinusoidal control input:

$$
\beta_{r}^{c}=3, H_{\beta_{r}}=0.3,
$$

- initial conditions:

$$
\begin{gathered}
x_{\beta_{20}}=\left[\begin{array}{l}
0 \\
0
\end{array}\right], \hat{x}_{0}^{c, 0}=\hat{x}_{0}^{c, 1}=\hat{x}_{0}^{c, 2}=\left[\begin{array}{l}
0.1 \\
0.1
\end{array}\right], \\
\hat{H}_{0}^{0}=\hat{H}_{0}^{1}=\hat{H}_{0}^{2}=\left[\begin{array}{cccc}
0.5 & 0 & 0 & 0 \\
0 & 0 & 0.5 & 0
\end{array}\right] .
\end{gathered}
$$

By iterating (15) thirty steps to obtain a satisfactory approximation of $\breve{X}_{\infty}^{i_{a} j_{a}}$, and according to (17), the corresponding approximation of residual-bounding zonotope $\stackrel{\circ}{R}_{\infty}^{i a j} j_{a}$ is computed. Eventually, all approximations of all relevant residual-bounding zonotopes are presented as

- for the interval observer 0 :

$$
\begin{aligned}
\stackrel{\circ}{R}_{\infty}^{10} & =[-0.8325,-0.2937], \\
\stackrel{\circ}{R}_{\infty}^{20} & =[-0.5570,-0.0778],
\end{aligned}
$$

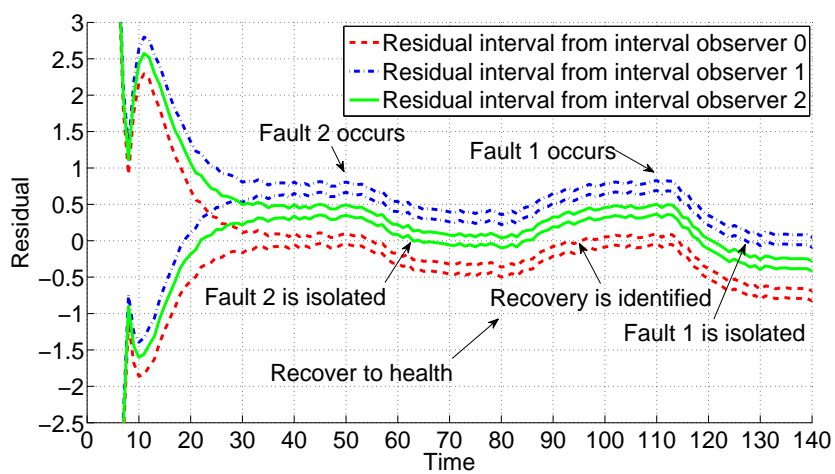

Fig. 1. The FDI of actuator faults

- for the interval observer 1 :

$$
\begin{aligned}
& \stackrel{\circ}{R}_{\infty}^{01}=[0.2889,0.8015], \\
& \stackrel{\circ}{R}_{\infty}^{21}=[0.0187,0.4567],
\end{aligned}
$$

- for the interval observer 2 :

$$
\begin{aligned}
\stackrel{\circ}{R}_{\infty}^{02} & =[0.0730,0.5255], \\
\stackrel{\circ}{R_{\infty}^{12}} & =[-0.4732,-0.0352],
\end{aligned}
$$

which shows that all the three considered actuator modes can satisfy the FDI conditions as indicated in (21). The simulation scenarios are considered as: from 0 to 50 the system is healthy, from 51 to 80 the second fault occurs, from 81 to 110 the system recovers to health and from 111 to 140 the first fault occurs.

The simulation results presented in Figure 1 show the effectiveness of this approach, where a transition appears when a fault occurs, which implies that the waiting time is necessary for the accurate FI.

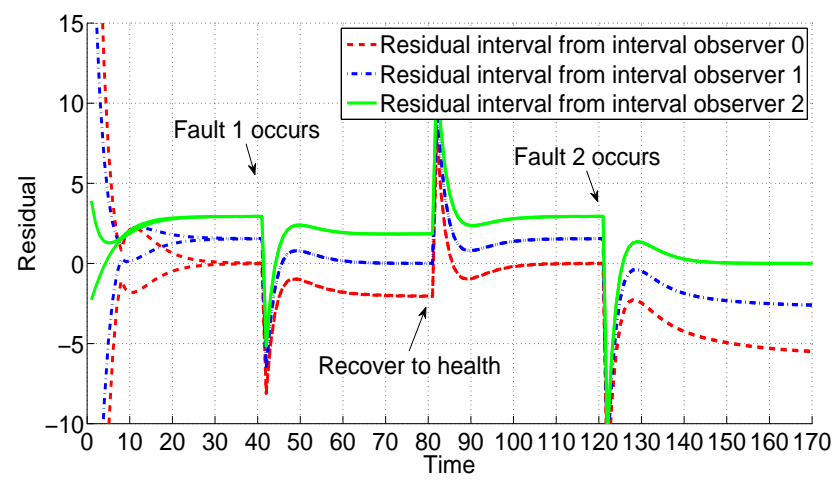

Fig. 2. The FDI of sensor faults

2) The Case of Sensor Faults: The original dynamics of the second blade subsystem characterized by Eqs.(4), (5) and (6) in [7] is used. The sensor faults are located in the second sensor described by Eq.(5) of [7]. In this simulation, the magnitudes of two sensor fault modes 1 and 2 are set as

$$
K_{1}=0.5, \quad K_{2}=0.05 .
$$


Noises and control inputs (all the other parameters respect those in the case of actuator faults) are given as

- measurement noises:

$$
\begin{aligned}
& \bar{\eta}_{\beta_{2}, m 1}=0.005, \quad \eta_{\beta_{2}, m 1}^{c}=1.5, \\
& \bar{\eta}_{\beta_{2}, m 2}=0.005, \quad \eta_{\beta_{2}, m 2}^{c}=1.5 .
\end{aligned}
$$

- sinusoidal control input:

$$
\beta_{r}^{c}=15, H_{\beta_{r}}=0.03
$$

Similarly, by iterating the corresponding bounding zonotops like (15) 50 steps, one computes the approximations of all relevant residual-bounding zonotopes

- for the interval observer 0 :

$$
\begin{aligned}
& \stackrel{\circ}{R}_{\infty}^{10}=[-10.400,-10.249], \\
& \stackrel{\circ}{R}_{\infty}^{20}=[-229.38,-227.53],
\end{aligned}
$$

- for the interval observer 1 :

$$
\begin{aligned}
\stackrel{\circ}{R}_{\infty}^{01} & =[1.2694,1.5076], \\
\stackrel{\circ}{R}_{\infty}^{21} & =[-0.9769,-0.9292],
\end{aligned}
$$

- for the interval observer 2 :

$$
\begin{aligned}
\stackrel{\circ}{R}_{\infty}^{02} & =[0.8013,4.4751], \\
\stackrel{\circ}{R}_{\infty}^{12} & =[-4.2844,-3.2450],
\end{aligned}
$$

which satisfy the corresponding guaranteed FDI conditions like (21).

Similarly, one sets simulation scenarios: from 0 to 40 the system is healthy, from 41 to 80 the first fault occurs, from 81 to 120 the system recovers to health and from 121 to 170 the second fault occurs. The simulation results presented in Figure 2 and Figure 3 illustrate the effectiveness of the method for sensor FDI.

\section{CONCLUSIONS}

This paper proposes an interval observer-based guaranteed FDI approach by using a bank of interval observers. For guaranteed FDI, a set of FDI conditions are established by analyzing the limit sets connected with invariant set notions. The advantage of the approach is that it can precheck whether the faults are detectable and isolable without the need of guaranteeing that residual zonotopes predicted by all the interval observers are separatable from each other. The following research is to explore ways of further reducing the conservativeness of FDI conditions for sensor faults.

\section{ACKNOWLEDGEMENTS}

The work of F. Xu, V. Puig and C. Ocampo-Martinez has been supported by the Spanish research project WATMAN (CICYT DPI2009-13744) of the Science and Technology Ministry and by the DGR of Generalitat de Catalunya (SAC group Ref. 2009/SGR/1491), and by European Comission through contract i-Sense (FP7-ICT-2009-6-270428). F. $\mathrm{Xu}$ is also supported by the China Scholarship Council (File No.2011629170). Acknowledgements to Juan Manuel Grosso for significant discussions.

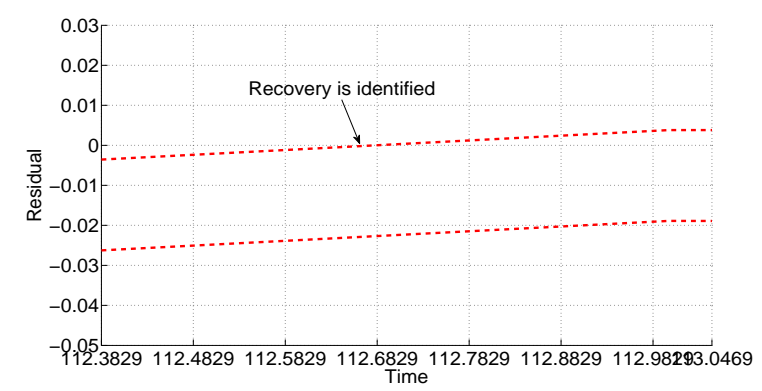

(a) Observer 0

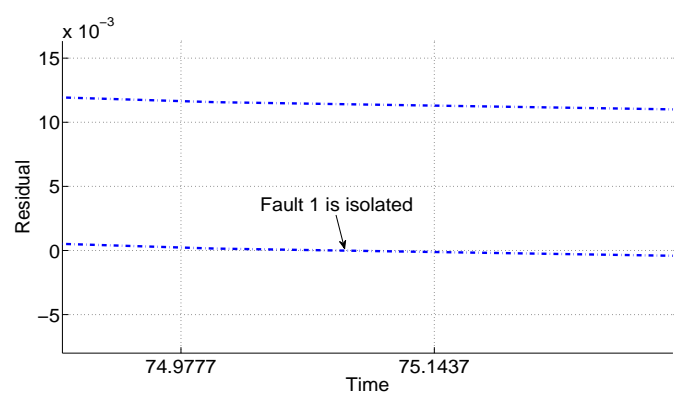

(b) Observer 1

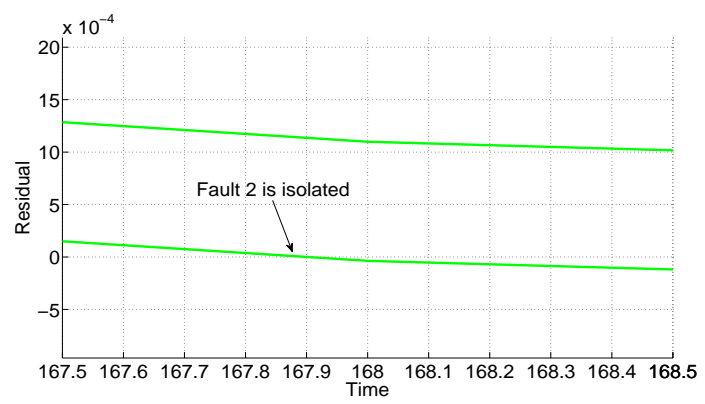

(c) Observer 2

Fig. 3. The scaling-up of the Fig.2

\section{REFERENCES}

[1] T. Alamo, J.M. Bravo, and E.F. Camacho. Guaranteed state estimation by zonotopes. In Decision and Control, 2003. Proceedings. 42nd IEEE Conference on, pages 5831 - 5836, Maui, Hawaii, USA, December 2003.

[2] P. Guerra, V. Puig, and M. Witczak. Robust fault detection with unknown-input interval observers using zonotopes. In Proceedings of the 17th World Congress, The International Federation of Automatic Control, COEX, Seoul, South Korea, July 2008.

[3] J. Meseguer, V. Puig, and T. Escobet. Robust fault detection linear interval observers avoiding the wrapping effect. In Proceedings of the 17th World Congress, The International Federation of Automatic Control, COEX, Seoul, South Korea, July 2008.

[4] S. Olaru, J.A. De Doná, M.M. Seron, and F. Stoican. Positive invariant sets for fault tolerant multisensor control schemes. International Journal of Control, 83(12):2622-2640, 2010.

[5] V. Puig, J. Quevedo, T. Escobet, and A. Stancu. Passive robust fault detection using linear interval observers. In IFAC Safe Process, Washington, USA, 2003.

[6] P. Rosa. Multiple-Model Adaptive Control of Uncertain LPV Systems. $\mathrm{PhD}$ thesis, Electrical and Computer Engineering, Instituto Superior Técnico, Portugal, 2011.

[7] F. Stoican, C.F. Raduinea, and S. Olaru. Adaptation of set theoretic methods to the fault detection of a wind turbine benchmark. In Proceedings of the 18th IFAC World Congress, pages 8322-8327, Milano, Italy, 28 August-2 September 2011. 